\title{
Inverse approach for estimating boundary properties in a transient fin problem
}

\author{
N GNANASEKARAN* and S BALAJI \\ Department of Mechanical Engineering, National Institute of Technology Karnataka, Surathkal, India \\ e-mail: gnanasekaran@nitk.edu.in
}

MS received 30 June 2017; revised 13 November 2017; accepted 11 December 2017; published online 22 June 2018

\begin{abstract}
A solution methodology is proposed for an inverse estimation of boundary conditions from the knowledge of transient temperature data. A forward model based on prevalent time-dependent heat conduction fin equation is solved using a fully implicit finite volume method. First, the inverse model is formulated and accomplished for time-invariant heat flux at the fin base, and later extended to transient heat flux, base temperature and average heat transfer coefficient. Secondly, the Nusselt number is then replaced with Rayleigh number in the forward model to realistically estimate the base temperature, which varies with respect to time, based on in-house transient fin heat transfer experiments. This scenario further corroborates the validation of the proposed inverse approach. The experimental set-up consists of a mild steel $250 \times 150 \times 6 \mathrm{~mm}^{3}$ fin mounted centrally on an aluminium base $250 \times 150 \times 8 \mathrm{~mm}^{3}$ plate. The base is attached to an electrical heater and insulated with glass-wool to prevent heat loss to surroundings. Five calibrated K-type thermocouples are used to measure temperature along the fin. The functional form of the unknown parameters is not known beforehand; sensitivity studies are performed to determine suitability of the estimation and location of sensors for the inverse approach. Conjugate gradient method with adjoint equation is chosen as the inverse technique and the study is performed as a numerical optimization; subsequently, the estimates show satisfactory results.
\end{abstract}

Keyword. Inverse; estimation; conjugate gradient; finite volume; sensitivity.

\section{Introduction}

Conventional methods in the field of heat transfer are useful to obtain the temperature distribution of the thermal system by incorporating necessary information. The knowledge of the thermal parameters aids the design and development of efficient heat exchange systems. However, to improve the heat transfer phenomena there are always some limitations associated with the conventional measurement techniques such as boundary conditions, initial conditions and thermophysical parameters. In such situations, the inverse heat transfer techniques play a crucial role in the estimation of unknown parameters based on the available data at the accessible locations. Hence, many researchers now rely on determination of unknown parameters based on parameter and function estimation methods.

The general overview of an inverse problem is to guess the unknown parameter that is under investigation and obtain the solution to the forward model heat transfer problem [1]. Once the forward model is solved for the assumed value of input, the next step is to compute the error between the simulated and experimental temperatures. If

*For correspondence the error is within the limit, the initial guess is the solution to the inverse problem; otherwise, the process is repeated until the condition is satisfied. The main objective of the inverse algorithms is to obtain the solution in the least possible time, imposing the minimal computational penalties. Any method, be it gradient or probabilistic approach, essentially performs a similar function (see figure 1). The classification of direct and inverse problems based on temperature time history has extensively been dealt with in [2], where it is proposed that the heat flux is more difficult to estimate than the surface temperature. Also, one can see fundamental understanding about regularization of inverse heat conduction problems (IHCPs) in [3]. Beck et al [4] compared several methods for the estimation of surface heat flux using transient temperature measurements inside a heat-conducting body. Jarny et al [5] proposed the estimation of the heat flux/temperature, space-dependent thermal conductivity and heat capacity from the knowledge of transient temperature. The approach has no a priori information about the unknown function to be determined. When one or more measured temperature histories of heat conduction space are available, the goal is then to estimate the unknown function or parameter appearing in the mathematical model [6]. A combination of boundary 


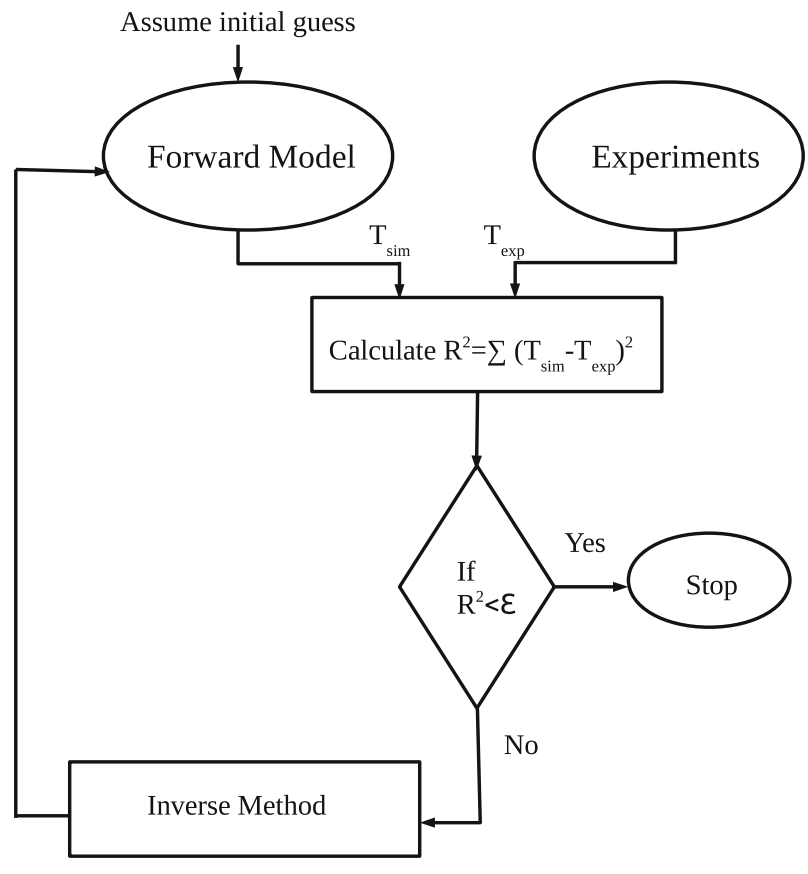

Figure 1. Overview of inverse estimation.

element method and an iterative-regularization-based inverse algorithm was used to solve the IHCP to estimate the transient boundary temperatures [7]. The implementation of three different versions of the conjugate gradient method (CGM), viz. Fletcher-Reeves, Polak-Ribiere and Powell-Beale versions, is discussed in [8]. Comparisons are performed for test cases involving different numbers of sensors, levels of measurement errors and initial guesses used for the iterative procedure. A three-dimensional transient heat conduction problem was solved using a commercial code and the CGM was applied to estimate the unknown boundary heat flux [9]. A simplified CGM is proposed in seeking shapes of inner structures for thermal data measured on outer surfaces of a solid body [10]. In the inverse analysis, the accuracy between simulated exact and inexact measurements of temperature at interior locations of pin fins was examined by the CGM [11]. Heat flux is estimated for different distributions for an inverse hyperbolic heat conduction problem in [12]. A two-dimensional inverse analysis is performed for functionally graded materials to estimate unknown transient surface heat flux; conjugate gradient with adjoint problem is used [13]. Temperature and heat flux on inaccessible surfaces are estimated for an IHCP based on the measured temperature and/or heat flux on accessible surfaces [14]. An IHCP of estimating the transient heat flux, applied on part of the boundary of an irregular region, was proposed; also, the solutions include the effects of noisy data and position of measured temperature [15]. Estimation of time- and spacedependent convection heat transfer coefficient of an annular fin is applied with the help of CGM while knowing the temperature or strain history at the measuring positions of the fin [16]. Determination of both the location and the time-varying strength of point heat sources within the body is presented using a combination of numerical and experimental approaches [17]. The convection heat transfer coefficient between the fin and the ambient fluid has been successfully computed when the thermal and strain fields are coupled and thus the estimation proved to be excellent for all the test data considered [18]. [19] Numerical results are presented for several benchmark problems with both exact and noisy data for the estimation for identifying the Robin coefficient based on boundary measurements. Wang et al [20] proposed an improved CGM for a three-dimensional parabolic partial differential equation to reconstruct the heat flux and source term based on the surface temperature measurements. Cheng et al [21] proposed a transient IHCP considering variations in material properties with respect to temperature and used CGM to recover the unknown heat flux based on the outer temperatures of the supersonic combustor. A combination of singular value decomposition method and CGM is proposed to improve the inverse solution [22]. [23] used a one-dimensional finite-volume-method-based transient inverse heat transfer model to estimate total heat flux from the cooling curves obtained using experiments.

Upon reviewing the literature on the estimation of boundary parameters and surface heat transfer coefficient based on inverse heat conduction approach, the powerful CGM even now proves to be an ardent option for the researchers [24]; nevertheless, the estimation of unknown parameters based on approximate fin model has not been addressed adequately with the aid of experimental investigation. As a result, an effort is attempted on an approximate heat transfer model, describing the heat exchange through an extended surface to estimate the unknown parameters such as heat flux, surface temperature at the boundary and also the heat transfer coefficient using the CGM and finally, incorporating the engineering knowledge for the validation of the proposed method for the actual temperature measurement based on fin experiments.

\section{Inverse problem}

The inverse problem is usually associated with the estimation of the unknown parameters based on the measurement taken by sensors located at known accessible locations in the domain. The problem is then solved as a minimization of the following residual function [25]:

$$
S[P(t)]=\sum_{i=1}^{M} \int_{t=0}^{t_{f}}\left\{Y_{i}\left(x_{m}, t\right)-T_{i}\left[x_{m}, t ; P(t)\right]\right\}^{2} d t
$$

where $i=1,2, \ldots, M$ is the number of sensors and $P(t)$ is the unknown parameter $\left(q, T_{b}\right.$ or $\left.h\right) . Y_{i}\left(x_{m}, t\right)$ is the measured temperature at the location $x=x_{m}$ at any given time 
$t$, and $T_{i}\left[x_{m}, t ; P(t)\right]$ is the estimated temperature obtained by solving the direct problem using an assumed value of the unknown parameter.

\section{Forward models}

A schematic diagram of the fin is presented in figure 2. Heat flux is applied at the boundary $(x=0)$, the rate of heat energy entering into the control volume is $q_{c o n d, x}$ and leaving out of the control volume is $q_{c o n d, x+\Delta x}$. The fin losing heat to the ambient is represented as $q_{c o n v}$. The timedependent one-dimensional conduction fin is considered to be the forward model. The governing equation is proposed as

$$
\frac{\partial^{2} T}{\partial x^{2}}-\frac{h p}{k A_{c}}\left(T(x, t)-T_{\infty}\right)=\frac{1}{\alpha} \frac{\partial T}{\partial t} .
$$

The boundary conditions are

$$
\begin{gathered}
-\left.k \frac{\partial T}{\partial x}\right|_{x=0}=q(t) \\
-\left.k \frac{\partial T}{\partial x}\right|_{x=L}=h\left(T_{(x=L, t)}-T_{\infty}\right) \\
T(x, 0)=T_{\infty}
\end{gathered}
$$

where $T(x, t)$ is the transient temperature in the fin, $p$ is the fin perimeter $(\mathrm{m}), A_{c}$ is the area of cross section of the fin $\left(\mathrm{m}^{2}\right), t$ is time in seconds (s), $h$ is the convective

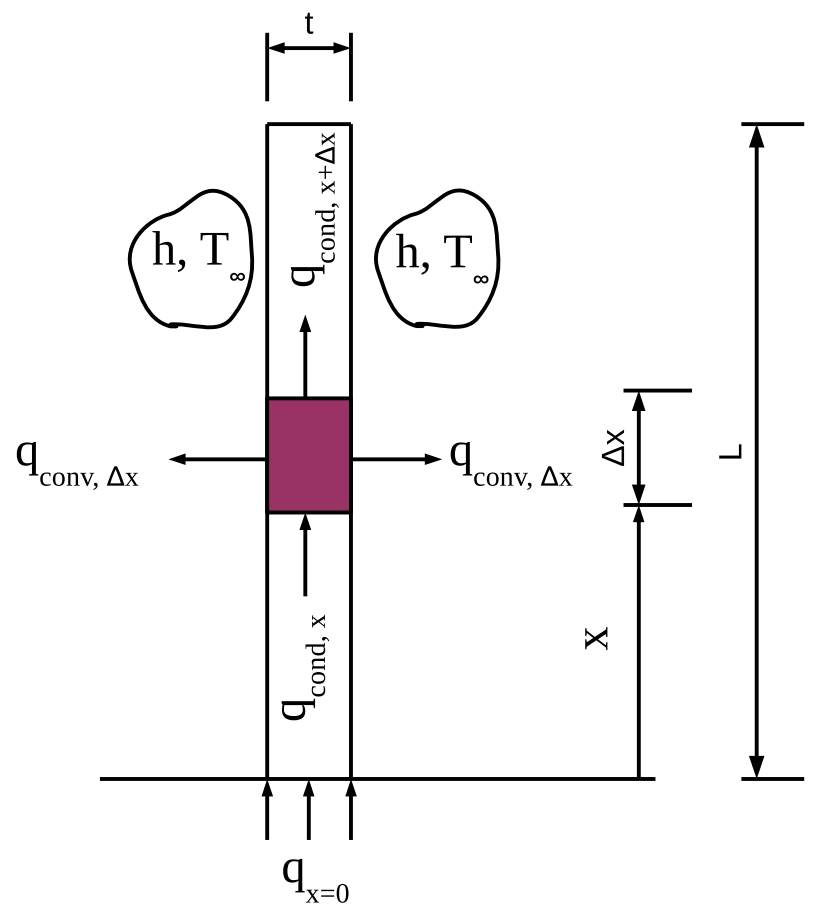

Figure 2. Schematic representation of the fin. heat transfer coefficient $\left(\mathrm{Wm}^{-2} \mathrm{~K}^{-1}\right), k$ is the thermal conductivity of fin material $\left(\mathrm{Wm}^{-1} \mathrm{~K}^{-1}\right)$ and $\alpha$ is the thermal diffusivity $\left(\mathrm{m}^{2} \mathrm{~s}^{-1}\right)$. The temperature measurements are taken in the $x$-direction by sensors placed at various locations from the base. The model is solved using a fully implicit finite-volume method and grid independence study is performed in order to obtain proper convergence.

\subsection{Forward problem for the heat flux}

3.1a The sensitivity problem: The solution to the direct model is regarded as an optimization problem to determine the value of unknown $q(t)$ to minimize the function $S$ defined by Eq. (1). The sensitivity function $\Delta T(x, t)$ is defined as the solution of the sensitivity problem, which is also the directional derivative of temperature $T(x, t)$ in the direction of perturbation of the unknown parameter. The problem can be solved by considering that the temperature $T(x, t)$ undergoes a perturbation of $\Delta T(x, t)$ when the heat flux $q(t)$ is perturbed by $\Delta q(t)$. Thus, by replacing $T(x, t)$ with $T(x, t)+\Delta T(x, t)$ and $q(t)$ with $q(t)+\Delta q(t)$ in the direct problem Eqs. (2)-(5) and subtracting the original direct problem equation from the resulting expressions, we obtain the following sensitivity problem:

$$
\begin{gathered}
\frac{\partial^{2} \Delta T}{\partial x^{2}}-\frac{h p}{k A_{c}}(\Delta T(x, t))=\frac{1}{\alpha} \frac{\partial \Delta T}{\partial t} \\
-\left.k \frac{\partial \Delta T}{\partial x}\right|_{x=0}=\Delta q(t) \\
-\left.k \frac{\partial \Delta T}{\partial x}\right|_{x=L}=h\left(\Delta T_{(x=L, t)}\right) \\
\Delta T(x, 0)=0 .
\end{gathered}
$$

$3.1 \mathrm{~b}$ Adjoint problem and gradient equation: In order to obtain the adjoint problem, Eq. (2) is multiplied by the Lagrange multiplier $\lambda(\mathrm{x}, \mathrm{t})$ and the resulting expressions are integrated over the space and time domains. This is then added to the right-hand side of Eq. (1) to yield the following expression:

$$
\begin{aligned}
& S[q(t)]=\int_{t=0}^{t_{f}} \sum_{i=1}^{M}\left\{Y(t)-T\left[x_{m}, t ; q(t)\right]\right\}^{2} \\
& +\int_{x=0}^{L} \int_{t=0}^{t_{f}} \lambda(x, t)\left[\frac{\partial^{2} T}{\partial x^{2}}-\frac{h p}{k A_{c}}\left(T-T_{\infty}\right)-\frac{1}{\alpha} \frac{\partial T}{\partial t}\right] d t d x .
\end{aligned}
$$

The variation $\Delta S$ is obtained by perturbing $T(x, t)$ by $\Delta T(x, t)$ in Eq. (10) and subtracting it from Eq. (10). Then we obtain 


$$
\begin{aligned}
& \Delta S[q(t)]=\int_{t=0}^{t_{f}} \int_{x=0}^{L} 2\left\{T\left[x_{m} ; q(t)\right]\right. \\
& \quad-Y(t)\} \Delta T(x, t) \delta\left(x-x_{\text {meas }}\right) d x d t \\
& \quad+\int_{t=0}^{t_{f}} \int_{x=0}^{L} \lambda(x, t)\left[\frac{\partial^{2} \Delta T}{\partial x^{2}}-\frac{h p}{k A_{c}} \Delta T-\frac{1}{\alpha} \frac{\partial \Delta T}{\partial t}\right] d t d x
\end{aligned}
$$

where $\delta($.$) is the Dirac Delta function. Utilizing the$ boundary and initial conditions of the sensitivity problem, the double integral on the right-hand side is evaluated by parts, and then $\Delta S$ is set to zero. After certain mathematical manipulations and by setting $\Delta T$ as zero, the following problem is obtained:

$$
\begin{gathered}
\frac{\partial^{2} \lambda(x, t)}{\partial x^{2}}-\frac{h p \lambda(x, t)}{k A_{c}}+\frac{1}{\alpha} \frac{\partial \lambda(x, t)}{d t} \\
+2\left(T\left(x_{m}, t\right)-Y\left(x_{m}, t\right)\right) \delta\left(x-x_{m}\right)=0 \\
\left.\frac{\partial \lambda(x, t)}{\partial x}\right|_{x=0}=0 \\
-\left.k \frac{\partial \lambda(x, t)}{\partial x}\right|_{x=L}=h \lambda_{x=L, t}, \\
\lambda\left(x, t_{f}\right)=0 .
\end{gathered}
$$

Finally, the integral term is expressed as follows:

$$
\Delta S[q(t)]=\int_{t=0}^{t_{f}} \lambda(0, t)(\Delta q(t) / k) d t .
$$

From the definition of the gradient for this technique, we have

$$
\Delta S[q(t)]=\int_{t=0}^{t_{f}} \nabla S[q(t)] \Delta q(t) d t
$$

where $\nabla S$ is the gradient of the functional S. A comparison of Eqs. (16)-(17) yields the following gradient equation:

$$
\nabla S[q(t)]=\lambda(0, t) / k .
$$

The adjoint problem as defined by Eqs. (12)-(15) is different from a conventional initial value problem in that the final time $t=t_{f}$ is specified instead of the customary initial time step. However, this problem can be converted into an initial value problem by transforming the time variable as $\tau=t_{f}-t$.

\subsection{Forward problem for the base temperature}

This case deals with the fin subjected to transient temperature at the base. The governing equation for this case is given in Eq. (2). The boundary conditions are the same except

$$
\left.T\right|_{x=0}=T_{b}
$$

3.2a Sensitivity problem

$$
\begin{gathered}
\left.\Delta T\right|_{x=0}=\Delta T_{b}(t), \\
-\left.k \frac{\partial \Delta T}{\partial x}\right|_{x=L}=h\left(\Delta T_{(x=L, t)}\right), \\
\Delta T(x, 0)=0 .
\end{gathered}
$$

3.2b Adjoint problem and gradient equation: The governing equation of the adjoint problem is the same as Eq. (12). The boundary conditions for this model are

$$
\begin{gathered}
\lambda(x=0, t)=0 \\
-\left.k \frac{\partial \lambda(x, t)}{\partial x}\right|_{x=L}=h\left(\lambda_{(x=L, t)}\right) \\
\lambda\left(x, t_{f}\right)=0 .
\end{gathered}
$$

Comparing the definition of gradient term with obtained expressions, the following equations are obtained:

$$
\Delta S\left[T_{b}(t)\right]=\int_{t=0}^{t_{f}} \nabla S\left[T_{b}(t)\right] \Delta T d t=\left.\int_{t=0}^{t_{f}} \frac{\partial \lambda(x, t)}{\partial x}\right|_{x=0} \Delta T d t
$$

Hence

$$
\nabla S\left[T_{b}(t)\right]=\left.\frac{\partial \lambda(x, t)}{\partial x}\right|_{x=0}
$$

\subsection{Forward problem for the heat transfer coefficient}

The governing equation for the forward problem is the same as Eqs. (2)-(5), but the unknown parameter is the heat transfer coefficient. For an assumed heat transfer coefficient, the forward temperature solution is obtained by solving Eqs. (2)-(5). The sensitivity equations are given as follows:

$$
\begin{gathered}
\frac{\partial^{2} \Delta T}{\partial x^{2}}-\frac{h p}{k A_{c}}(\Delta T(x, t))-\frac{\Delta h p}{k A_{c}}\left(T(x, t)-T_{\infty}\right)=\frac{1}{\alpha} \frac{\partial \Delta T}{\partial t} \\
-\left.k \frac{\partial \Delta T}{\partial x}\right|_{x=0}=0 \\
-\left.k \frac{\partial \Delta T}{\partial x}\right|_{x=L}=0 \\
\Delta T(x, 0)=0
\end{gathered}
$$

The complexity associated with Eqs. (2)-(5) is the convective heat transfer coefficient appearing in both governing equation and the boundary condition; to resolve, this particular case is assumed to have insulated boundary at the fin tip. 
3.3a The adjoint and gradient equation: Owing to change in sensitivity model equation, the adjoint problem is suitably modified to incorporate the non-linearity:

$$
\begin{aligned}
\Delta S & {[h(t)]=\int_{t=0}^{t_{f}} \int_{x=0}^{L} 2\left\{T\left[x_{m} ; h(t)\right]\right.} \\
& -Y(t)\} \Delta T(x, t) \delta\left(x-x_{\text {meas }}\right) d x d t \\
& +\int_{t=0}^{t_{f}} \int_{x=0}^{L} \lambda(x, t)\left[\frac{\partial^{2} \Delta T}{\partial x^{2}}-\frac{h p}{k A_{c}} \Delta T-\frac{\Delta h p}{k A_{c}}\left(T(x, t)-T_{\infty}\right)\right. \\
& \left.-\frac{1}{\alpha} \frac{\partial \Delta T}{\partial t}\right] d t d x .
\end{aligned}
$$

Following the same sequence of steps in further derivation, the following model is obtained:

$$
\begin{gathered}
\frac{\partial^{2} \lambda(x, t)}{\partial x^{2}}-\frac{h p \lambda(x, t)}{k A_{c}}+\frac{1}{\alpha} \frac{\partial \lambda(x, t)}{d t} \\
+2\left(T\left(x_{m}, t\right)-Y\left(x_{m}, t\right)\right) \delta\left(x-x_{m}\right)=0, \\
\left.\frac{\partial \lambda(x, t)}{\partial x}\right|_{x=0}=0, \\
\left.\frac{\partial \lambda(x, t)}{\partial x}\right|_{x=L}=0, \\
\lambda\left(x, t_{f}\right)=0 .
\end{gathered}
$$

Finally, the integral term is expressed as follows:

$$
\Delta S[h(t)]=-\int_{t=0}^{t_{f}} \frac{p}{k A_{c}} \int_{x=0}^{L} \lambda\left(T-T_{\infty}\right) \Delta h d x d t .
$$

From the definition of the gradient for this technique, we have

$$
\Delta S[h(t)]=\int_{t=0}^{t_{f}} \nabla S[h(t)] \Delta h(t) d t
$$

where $\nabla S$ is the gradient of the functional $S$ :

$$
\nabla S[h(t)]=-\frac{p}{k A_{c}} \int_{x=0}^{L} \lambda\left(T-T_{\infty}\right) d x .
$$

The gradient term now takes an integral form, which adds to computational difficulties. If spatial variation of heat transfer coefficient was considered, the above expression would have been a double integral over space and time, which would have posed a significant challenge for efficient computation. Numerical integration by itself contains certain error. In addition, multiple integration amounts only compound the error, and increase the computational time, thereby rendering the selection of this technique pointless. Hence, estimation is performed only for average heat transfer coefficient over the fin.

\section{Solution methodology}

As discussed earlier, the CGM is used as the inverse technique. Initially, the model is solved for time-invariant heat flux based on simulated measurements. This is then extended to solve transient heat flux functions of various forms. This is followed by the estimation of base temperature and heat transfer coefficient. For each transient case, effect of number of sensors on accuracy is also studied. Once computational robustness is established, experimental results are used for parameter estimation.

\subsection{Iterative procedure}

The mathematical development of the inverse methodology contains three distinct problems defined by the direct, sensitivity and adjoint models for the computation of $T(x, t), \Delta T(x, t)$ and $\lambda(x, t)$, respectively. The experimental temperature $Y(t)$ is known from experiments. Assuming that the functions $T(x, t), \Delta T(x, t), \lambda(x, t)$ and $\nabla S$ are available at the $k^{\text {th }}$ iteration, an iterative sequence of steps are employed for proper selection of step size and direction of descent in going from iteration $k$ to iteration $k+1$. The parameters to be estimated at $(k+1)^{\text {th }}$ step can be calculated as

$$
P^{k+1}(t)=P^{k}(t)-\beta^{k} d^{k}(t)
$$

where $\beta^{k}$ is the search step size and $d^{k}(t)$ is direction of descent, defined as follows:

$$
d^{k}(t)=\nabla S\left[P^{k}(t)\right]+\gamma^{k} d^{k-1}(t) .
$$

Here, $\gamma^{k}$ is the coefficient of conjugation, determined from the Fletcher-Reeves equation [26] as

$$
\gamma^{k}=\frac{\int_{t=0}^{t_{f}}\left\{\nabla S\left[P^{k}(t)\right]\right\}^{2} d t}{\int_{t=0}^{t_{f}}\left\{\nabla S\left[P^{k-1}(t)\right]\right\}^{2} d t} \quad \text { with } \quad \gamma^{0}=0 .
$$

The step size $\beta^{k}$ can be evaluated from minimization of the functional $S[P(t)]$ and can be written as

$$
\beta^{k}=\frac{\sum_{i=1}^{M} \int_{t=0}^{t_{f}}\left\{T_{i}\left[x_{m}, t ; P^{k}(t)\right]-Y_{i}(t)\right\} \Delta T\left[x_{m}, t ; d^{k}(t)\right] d t}{\sum_{i=1}^{M} \int_{t=0}^{t_{f}}\left\{\Delta T_{i}\left[x_{m}, t ; d^{k}(t)\right]\right\}^{2} d t} .
$$

The stopping criterion based on the discrepancy principle is

$$
S(P(t))<\epsilon
$$

The tolerance $\epsilon$ is chosen so that smooth solutions are obtained with measurements containing random errors. 


\subsection{Computational algorithm}

1. Suppose an initial guess is available for the unknown parameter $P(t)$; set $k=0$ and then

2. Solve the direct problem and compute $T(x, t)$ based on $P^{k}(t)$;

3. check the stopping criterion Eq. (44); continue if not satisfied;

4. knowing $T\left(x_{\text {meas }}, t\right)$ and measured temperature $Y(t)$, solve the adjoint problem and compute $\lambda(x, t)$;

5 . knowing $\lambda(x, t)$, compute the gradient equation;

6. knowing the gradient, compute $\gamma^{k}$ from the FletcherReeves equation (42) and the direction of descent $d^{k}(t)$ from Eq. (41);

7. setting $\Delta P^{k}(t)=d^{k}(t)$, solve the sensitivity problem to obtain $\Delta T_{i}\left[x_{m}, t ; d^{k}(t)\right]$

8. knowing $\Delta T_{i}\left[x_{m}, t ; d^{k}(t)\right]$, compute the search step size from Eq. (43);

9. knowing the search step size $\beta^{k}$ and the direction of descent $d^{k}(t)$, compute the new estimate from Eq. (40) and return to step 2 .

The afore-mentioned process is applied to various cases detailed in the problem statement and the equations obtained are discussed in the subsequent sections.

\section{Description of experimental set-up}

An in-house fin heat transfer apparatus was fabricated for this study. The experimental set-up consists of a short rectangular steel fin of dimensions $250 \times 150 \times 6 \mathrm{~mm}^{3}$ mounted over an aluminium base of dimensions $250 \times 150 \times 8 \mathrm{~mm}^{3}$. A slot of $4 \mathrm{~mm}$ depth is machined centrally along base plate length, where the fin is mounted. Fin base is heated by an electrical heater of the same dimensions. Glass-wool is used beneath the heater to minimize the heat loss to ambient. Calibrated K-type thermocouples are used to record the temperatures of the fin and the base. A separate thermocouple is provided to measure the ambient temperature. Data acquisition is used to record the temperatures, monitored by LABVIEW software. A photographic view of the set-up is shown in figure 3. For the present study, multiple test voltages are considered starting from $40 \mathrm{~V}$ input.

\section{Results and discussion}

\subsection{Forward model validation}

The forward model is validated against the known analytical solution available in [27]. Eqs. (2)-(5) are solved for the constant base temperature boundary condition for the purpose of validation. The comparison between simulated and analytical temperatures is presented for two different locations shown in figure 4.

\subsection{Estimation of unknown parameters}

6.2a Constant heat flux estimation: Most of the inverse analysis is first tested with the simulated measurements in order to check the validity of the proposed methodology. Such an exercise is also followed here as well. Eqs. (2)-(5) are solved for the time-invariant heat flux for the fixed grid. The properties considered are reported in table 1 . The heat transfer coefficient " $h$ " is the critical parameter in this analysis and the value is assumed to be $5 \mathrm{Wm}^{-2} \mathrm{~K}^{-1}$, considering natural convection heat transfer. Simulations are then carried out with a single sensor placed at $0.1 \mathrm{~m}$. It is obvious that the measured temperature always contains

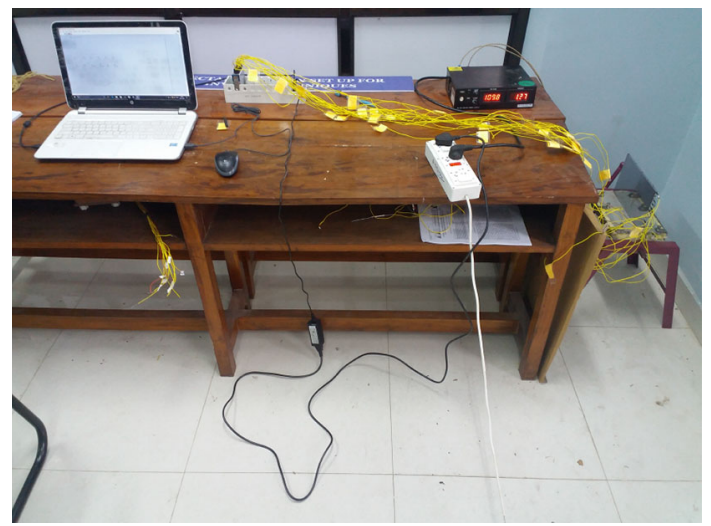

Figure 3. Photographic view of the experimental set-up.

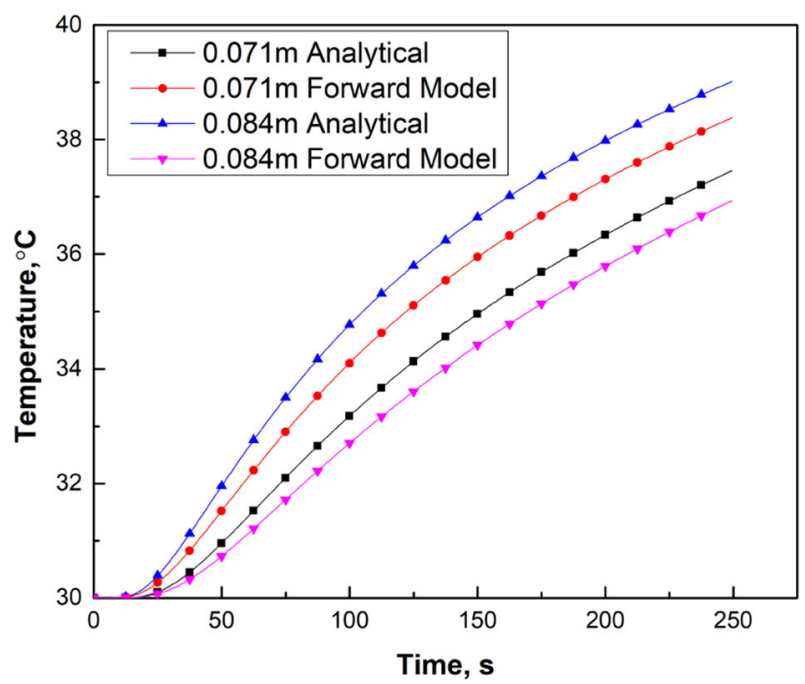

Figure 4. Forward model validation. 
Table 1. Parameters considered for simulation.

\begin{tabular}{lc}
\hline Parameters & Values \\
\hline Fin length & $0.15 \mathrm{~m}$ \\
Fin width & $0.25 \mathrm{~m}$ \\
Fin thickness & $0.006 \mathrm{~m}$ \\
Sensor locations & $0.05,0.075,0.1 \mathrm{~m}$ from the base \\
Fin material & Steel \\
Thermal conductivity $k$ & $36 \mathrm{Wm}^{-1} \mathrm{~K}^{-1}$ \\
Thermal diffusivity $\alpha$ & $1.172 \times 10^{-5} \mathrm{~m}^{2} \mathrm{~s}^{-1}$ \\
Ambient temperature & $30{ }^{\circ} \mathrm{C}$ \\
Grid size $d x$ & $0.5 \mathrm{~mm}$ \\
Time step $d t$ & $0.5 \mathrm{~s}$ \\
Final time $\mathrm{t}_{f}$ & $1000 \mathrm{~s}$ \\
\hline
\end{tabular}

inherent noise; therefore, as a representative case, $5 \%$ noise is added to the simulated temperatures and this is now treated as surrogate temperature measurement. Figure 5 shows the surrogate temperature time history without noise and figure 6 shows the temperature distribution with $5 \%$ noise. The error between the actual and estimated heat flux was observed to be $1.46 \%$.

Once the estimation is verified for the time-invariant flux, the methodology is then extended to solve transient heat flux. Here, two different heat flux profiles are considered: the actual and estimated heat flux are shown in figures 7 and 8 . The effect of multiple sensors has been studied for obtaining better accuracy of the estimate, which is shown in figure 9.

6.2b Estimation of base temperature: The idea of this proposed work is to fully exploit the fin model for the estimation of unknown parameters. In the earlier section, it has been shown that the unknown heat flux estimated for different profiles show satisfactory results even for noise added simulated measurements and hence, based on this background the numerical investigation is now ventured in to base temperature of the fin. To accomplish this, Eqs. (2)(5) along with Eq. (19) are solved to determine the unknown base temperature. Figures 10-12 show the estimation results and figure 12 clearly shows the increase in accuracy with the help of multiple sensors.

6.2c Estimation of heat transfer coefficient: The estimation of heat transfer coefficient plays a very crucial role in the inverse estimation process. The forward model is the same as Eq. (2) and the sensitivity model for the heat transfer coefficient (HTC) is proposed in Eq. (28). The estimation result is shown in figure 13. Note that there is an effect of the initial guess value on the estimation of the heat transfer coefficient. This effect is pronounced in the initial time-steps and gradually diminishes. This is tested with multiple initial guess values and a similar trend was observed each time. This can be attributed to the presence of a term containing " $h$ " in the sensitivity model equation. The value used in this equation would correspond to that of

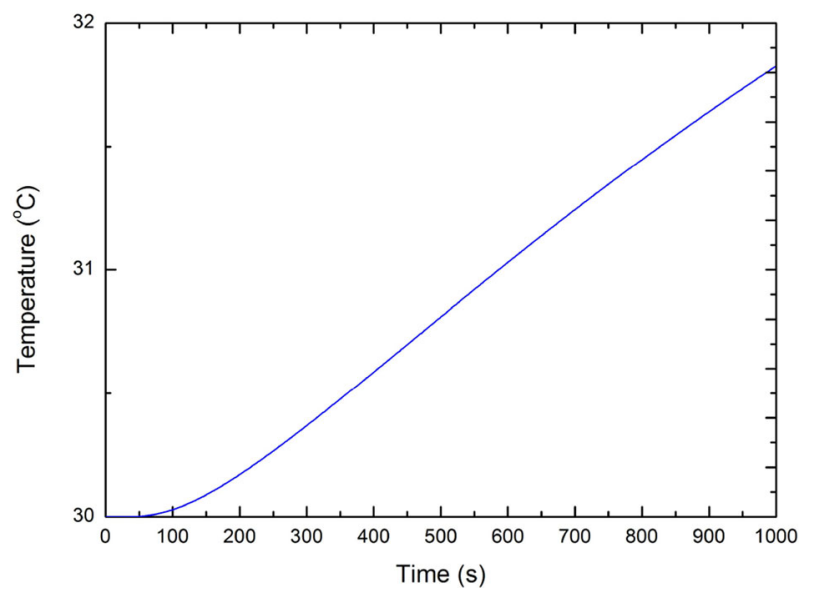

Figure 5. Temperature distribution for $q=1456 \mathrm{Wm}^{-2}$.

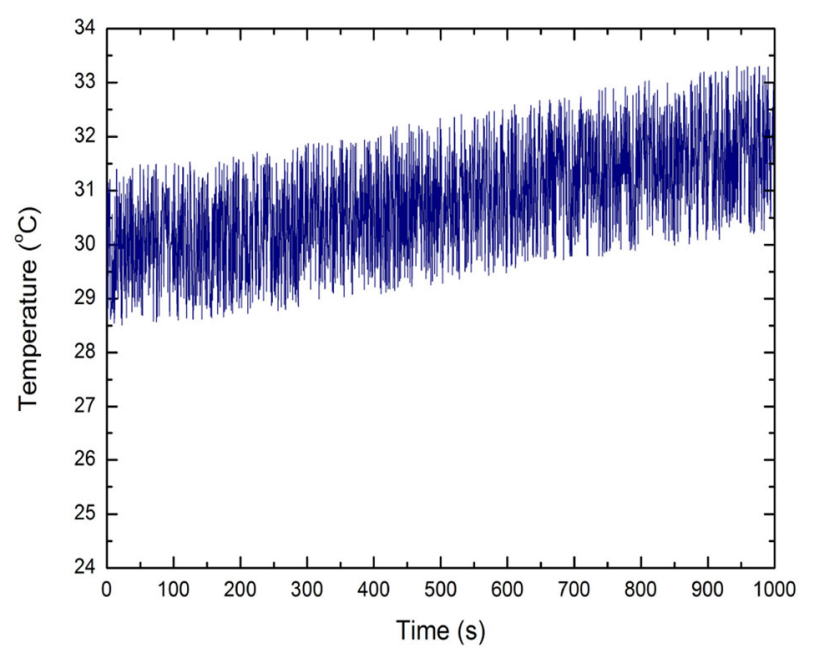

Figure 6. Temperature distribution with added noise of $5 \%$ for $q=1456 \mathrm{Wm}^{-2}$.

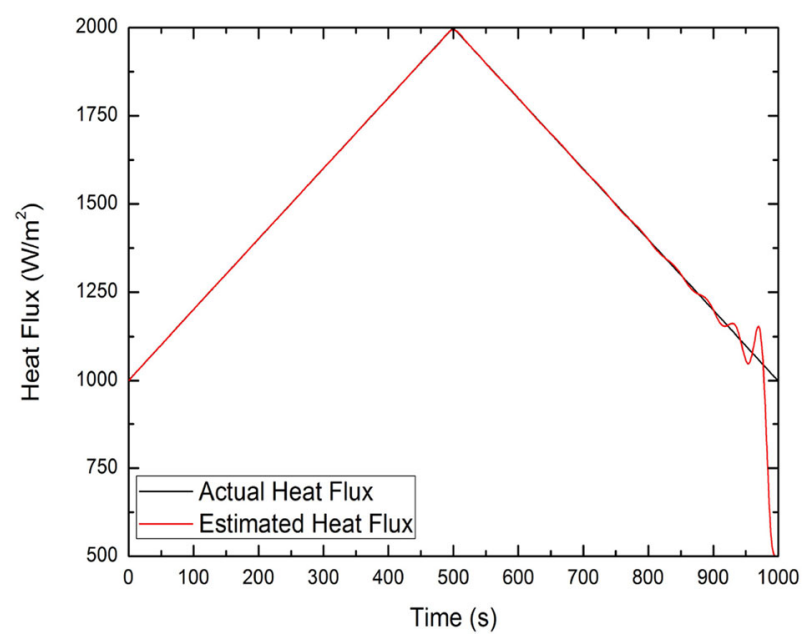

Figure 7. Estimation of heat flux for triangular profile. 


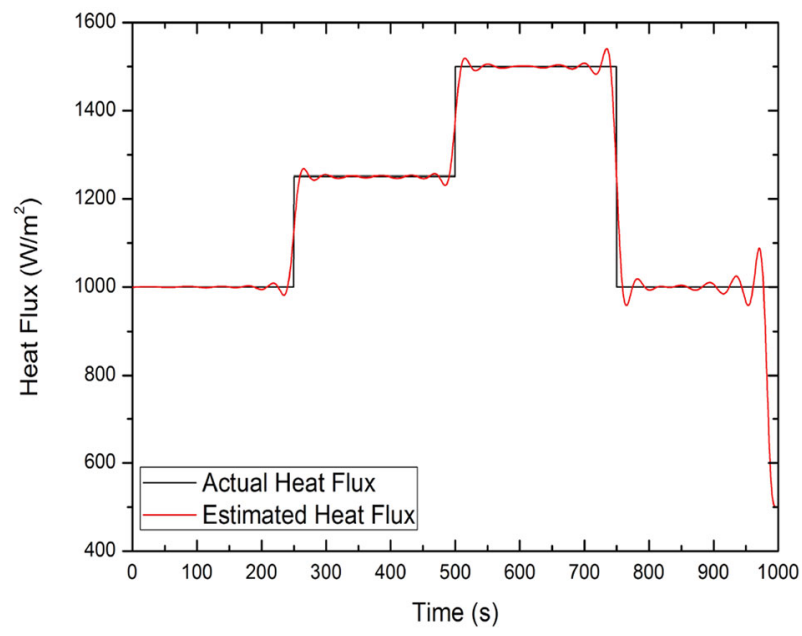

Figure 8. Estimation of step profile heat flux at base.

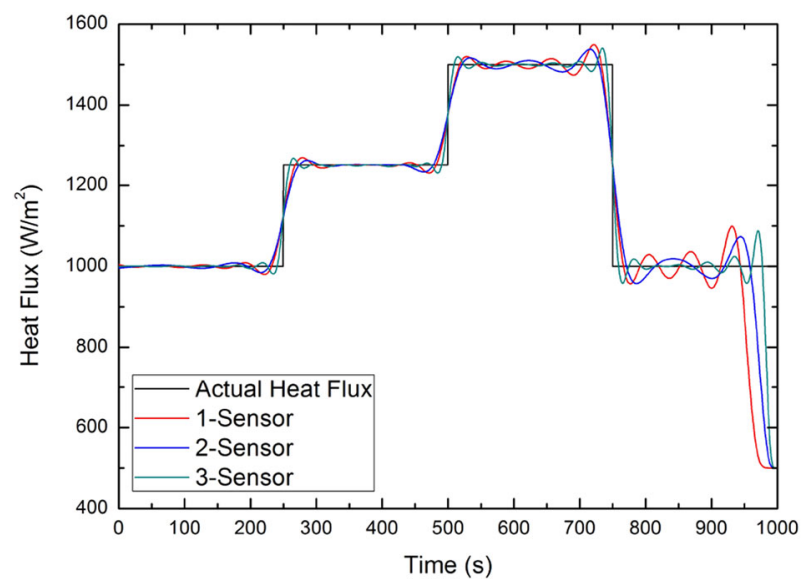

Figure 9. Effect of multiple sensors on heat flux estimation.

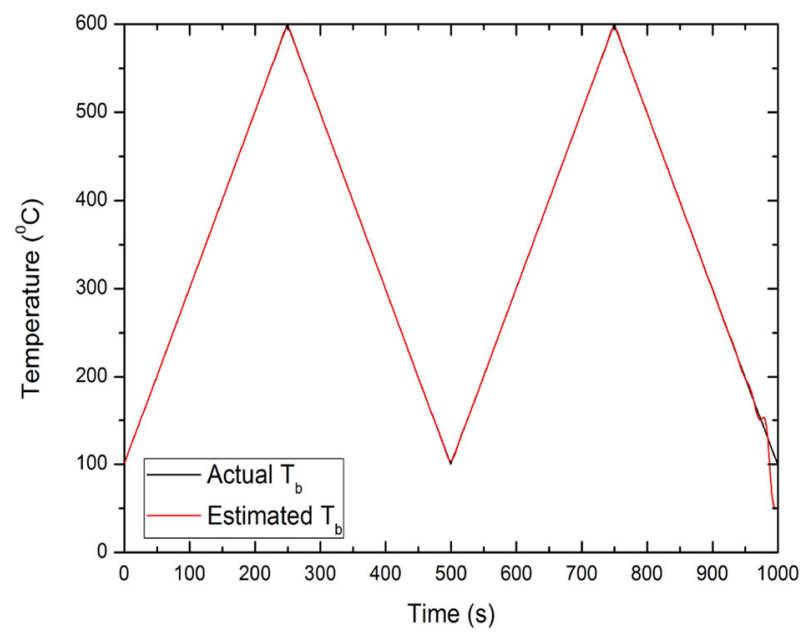

Figure 10. Estimation of base temperature for triangular profile.

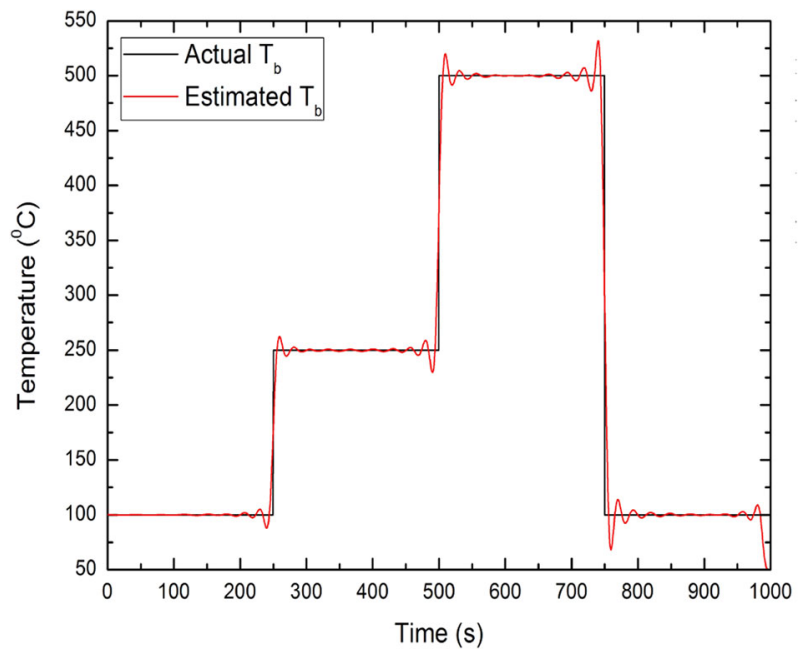

Figure 11. Estimation of base temperature for square profile.

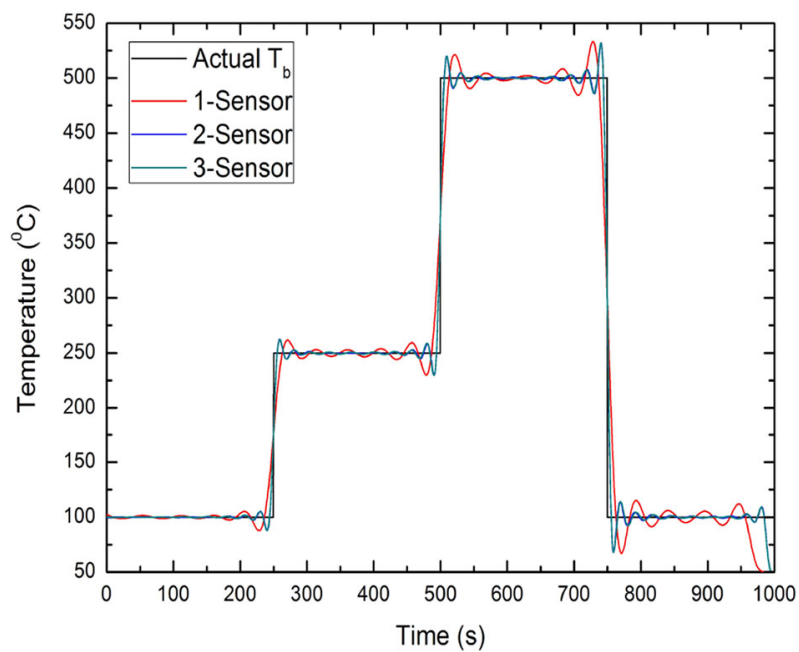

Figure 12. Effect of multiple sensors on base temperature estimation.

a previous iteration. Solution to the sensitivity model is used to compute the step size and subsequent value of unknown parameter for next iteration. This implies that the next iteration estimate is affected by the previous iteration estimate, and the error is carried forward. This error gradually decreases due to the iterative procedure, but the effects are still felt in the estimated profile. To mitigate this error, multiple initial guesses need to be considered. These iterations reveal an optimum starting point where there are less errors (see figure 13).

Also, note that in transient estimations, there is a deviation in predicted values near the end of the time-frame of observations. As $t$ approaches $t_{f}$, the solution deviates towards the initial guess value used for estimation. This is attributed to the presence of a null gradient for temperature at $t=t_{f}$ due to the observations and input ceasing. This 


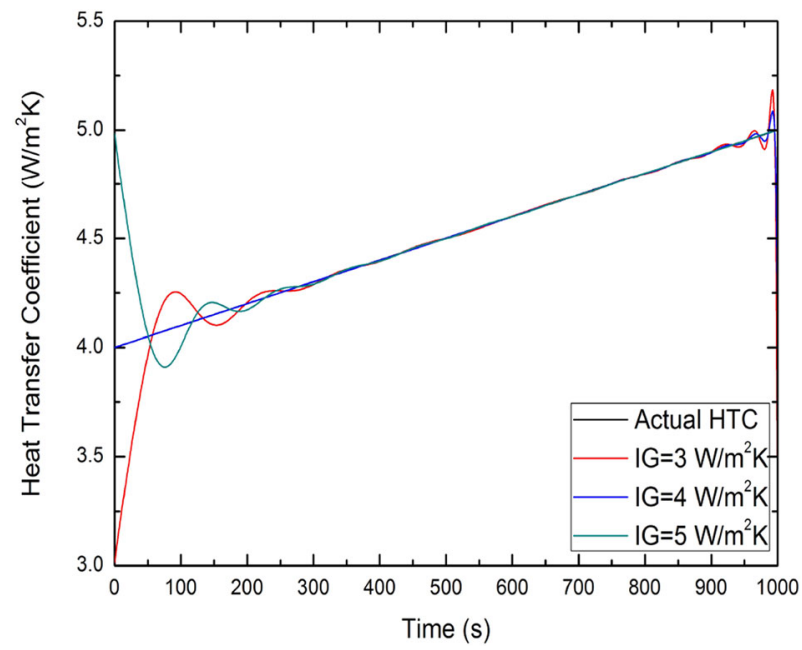

Figure 13. Effect of initial guess on the estimation of convective heat transfer coefficient.

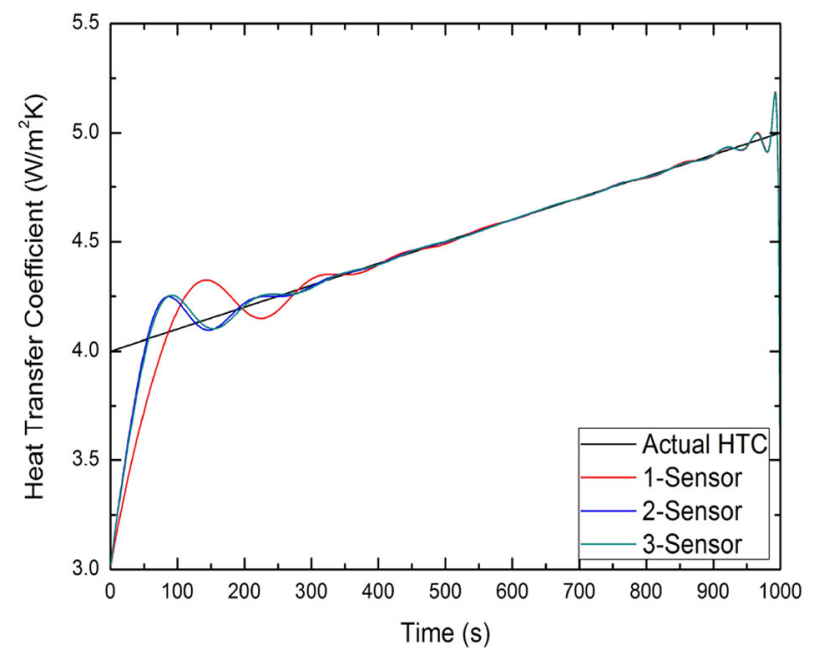

Figure 14. Effect of sensors on HTC estimation; initial guess $=3$ $\mathrm{Wm}^{-2} \mathrm{~K}^{-1}$.

affects the solution in the neighbourhood of $t_{f}$. To improve the solution in this region, the algorithm must be solved for a longer final time. Hence, for better accuracy, to estimate near $t_{f}=1000 \mathrm{~s}$, the solution must be obtained for $t_{f}>1000 \mathrm{~s}$. This, in turn, can cause the solution to deviate at other locations. Another method of mitigation is to use multiple sensors. A single sensor estimate is always less accurate than a multiple sensor estimate under similar conditions. It is evident from the results that the neighbourhood of deviations near $t_{f}$ is smaller for a three-sensor estimate as compared with a single-sensor estimate (figure 14). However, increasing the number of sensors leads to rise in number of terms in the objective function. This leads to a higher residual error in the system, thus preventing the residual from decreasing below a certain point.
This is evident in the plots with estimates from multiple sensors, where it can be observed that the three-sensor estimate line has less span of incorrect solution near the end. Hence, more sensors leads to increased accuracy towards the end, but there is a chance that residual value can increase. As the number of sensors increases, number of terms in objective function increases. This would mean that the error value will not decrease below a certain point.

\subsection{Experimental investigation}

To determine the applicability of CGM to experimental data, a sensitivity analysis is performed before attempting the estimation of unknown parameters. The forward model is incorporated with Nusselt number correlation $N u=$ $C R a^{n}$ as proposed in [28] and the governing equation now becomes

$$
\frac{\partial^{2} T}{\partial x^{2}}-\frac{C R a^{n} k_{f} p}{L k A_{c}}\left(T(x, t)-T_{\infty}\right)=\frac{1}{\alpha} \frac{\partial T}{\partial t}
$$

where $R a=\left(g \beta \Delta T L^{3}\right) / v \alpha$; the boundary conditions are

$$
\left.T\right|_{x=0}=q
$$

or

$$
\begin{gathered}
\left.T\right|_{x=0}=T_{b}(t), \\
-\left.k \frac{\partial T}{\partial x}\right|_{x=L}=h\left(T_{(x=L, t)}-T_{\infty}\right), \\
T(x, 0)=T_{\infty} .
\end{gathered}
$$

The values of $C$ and $n$ are considered as 0.59 and 0.25 , respectively. A higher value of the sensitivity coefficient indicates that there will be an appreciable change in temperature for small changes in the unknown parameters. This indicates that estimation will be fine-tuned and able to pick up small fluctuations in input. On the other side, a lower sensitivity coefficient would indicate an increased difficulty in reliable estimation of parameters under investigation. In the present study the parameters under consideration are the heat flux, base temperature and convective heat transfer coefficient. The inexpensive experimental set-up is aimed at determining the base temperature and not the heat flux, as the heater at the base provides a constant magnitude of heat flux. However, as with the most recent works based on isothermal plates [29], here too, the concept of Biot number is invoked for the aluminium base. For this, though the base plate undergoes conduction the thermocouples placed at different locations in the base plate recorded a temperature difference of only $0.5^{\circ} \mathrm{C}$; also, the Biot number calculated for the aluminium base was found to be less than 0.1 and this is more than sufficient to assume the base plate to be a lumped system. To make this stand clear, scaled sensitivity coefficients for all three parameters are plotted in figure 15 . 


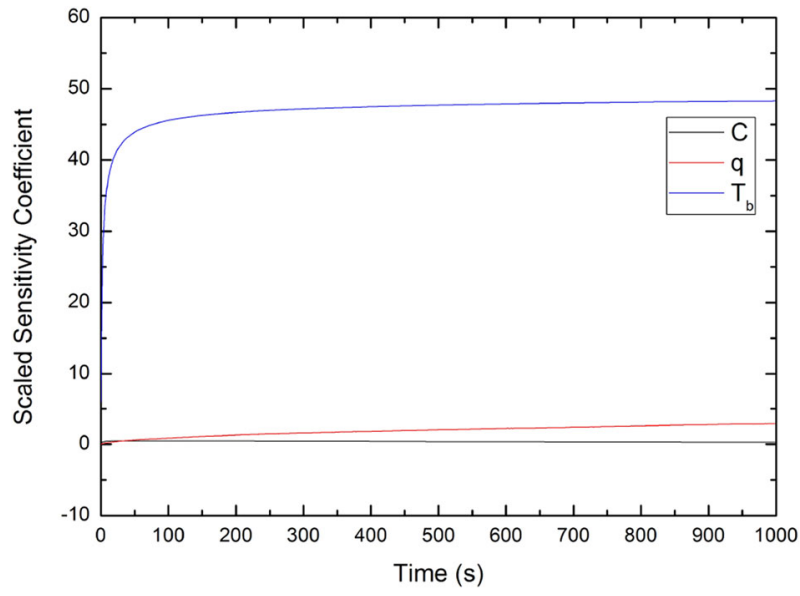

Figure 15. Scaled sensitivity coefficient.

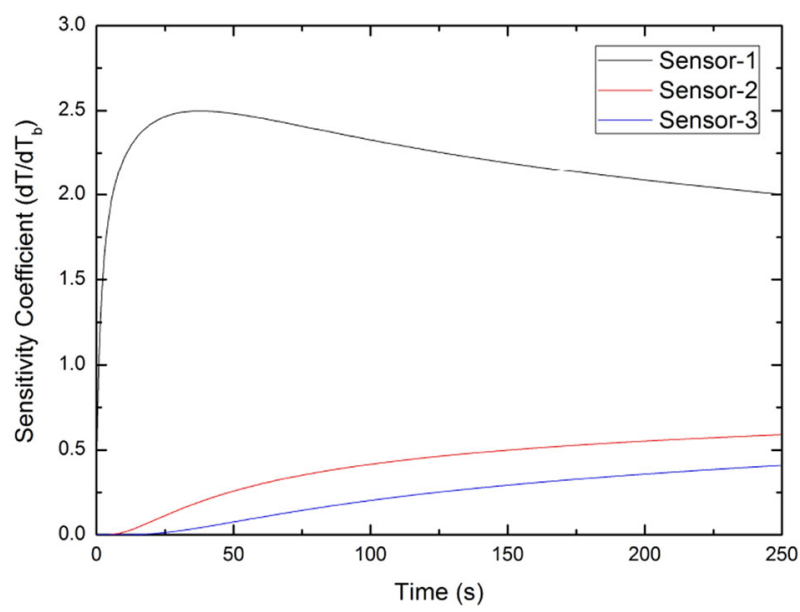

Figure 16. Effect of sensors for the estimation of $\mathrm{T}_{b}$.

Note that while $T_{b}$ has an appreciable sensitivity, the value is close to zero for other parameters. This implies that only $T_{b}$ can genuinely be estimated from the present model. Hence, the investigation is performed only for $T_{b}$.

The next step in the sensitivity study is to determine the optimum location of sensors on the fin for accurate estimation of unknown parameters. Sensitivity coefficient is plotted for sensors placed at different locations on the fin.

Figure 16 shows the effect of sensor location on the estimation of $T_{b}$. Sensor-1 is closest to the fin base while sensor- 3 is the farthest. Note that as the distance from base increases, the sensitivity coefficient decreases. This implies that sensors placed beyond location- 3 would see a further drop in sensitivity and hence do not contribute to the accuracy of the solution. The locations as determined by sensitivity study are presented in table 2. Estimation is performed for 50 iterations for each input case. Convergence residual is monitored and iterations are terminated
Table 2. Details of the locations of sensors.

\begin{tabular}{lc}
\hline Sensor & Location from fin base $(\mathrm{m})$ \\
\hline Sensor-1 & 0.006 \\
Sensor-2 & 0.045 \\
Sensor-3 & 0.071 \\
\hline
\end{tabular}

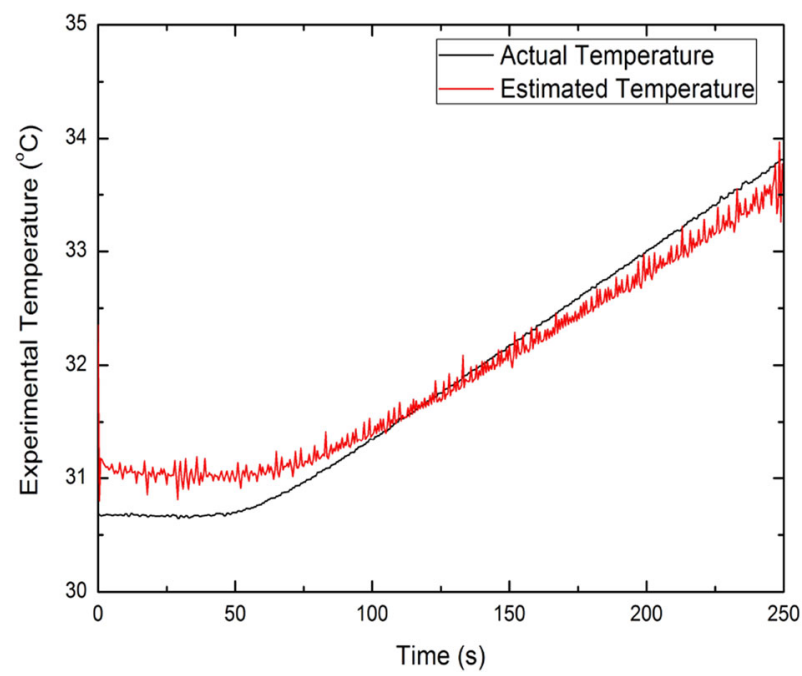

Figure 17. Estimation of $T_{b}$ for $40 \mathrm{~V}$.

accordingly. Data from all three sensors are used simultaneously for higher accuracy in the estimation. Figure 17 shows the estimation of the base temperature $T_{b}$ for the experimental temperature. Also, the corresponding objective function is shown in figure 18. An appreciable prediction of the estimated base temperature seen in figures 17 and 18, where the objective function is drastically

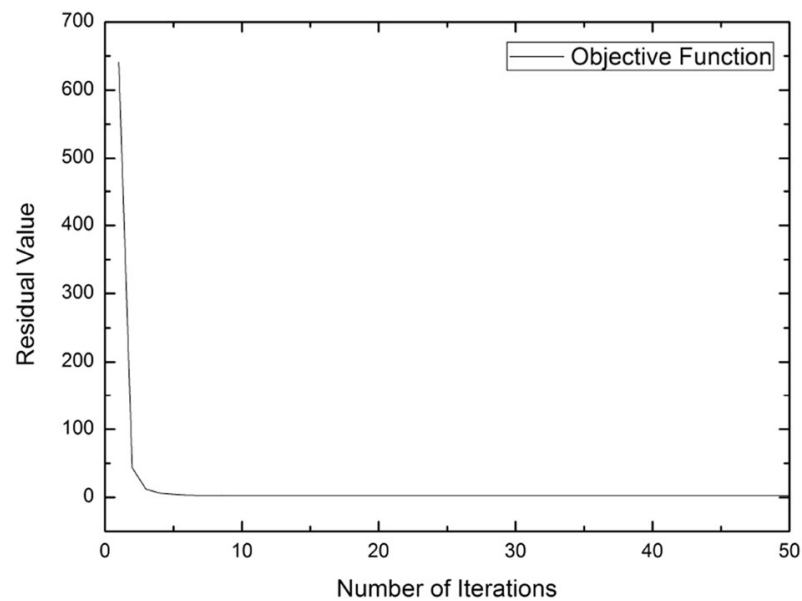

Figure 18. Residual of the objective function for $40 \mathrm{~V}$. 


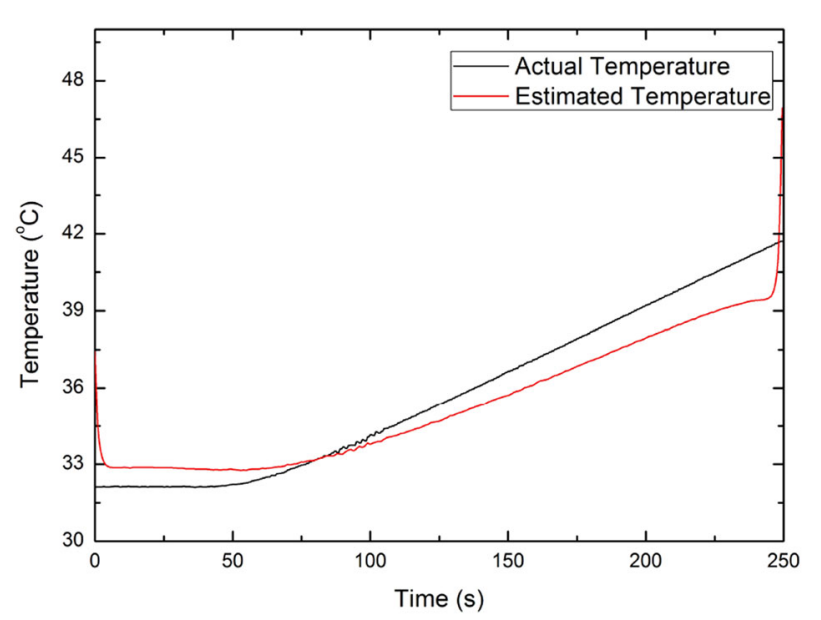

Figure 19. Estimation of $T_{b}$ for $70 \mathrm{~V}$.

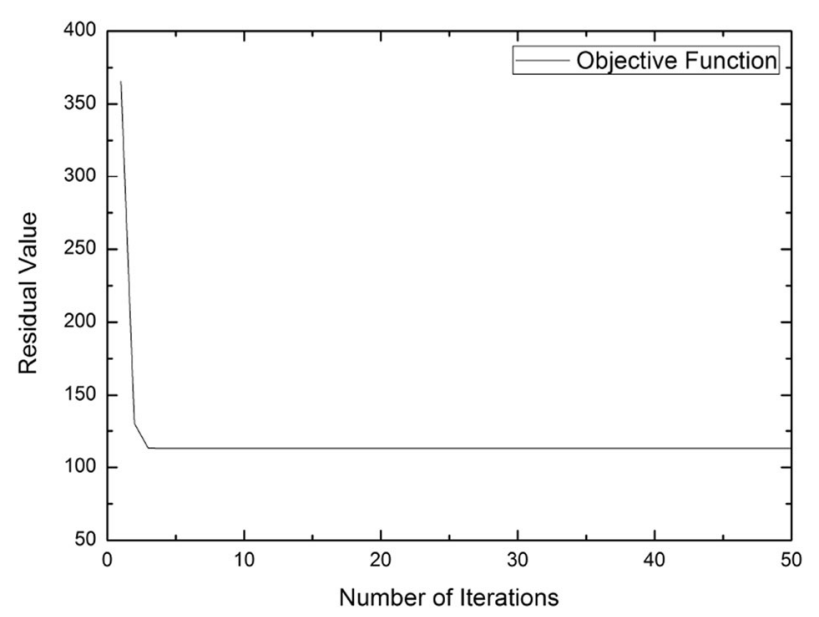

Figure 20. Residual of the objective function for $70 \mathrm{~V}$.

minimized well within a few iterations, motivated another attempt, this time with higher input. Such a result is shown in figures 19 and 20 and thus, the computational benefit by employing such a method is clearly revealed based on the residual error.

\section{Conclusion}

A method to determine the unknown parameters associated with fin heat transfer was discussed and implemented in this work. The methodology employed comprises two parts: computational modelling, testing and validation, and experimental investigation into parameter recovery based on in-house experiments. The estimation was performed by solving an inverse heat conduction fin problem (IHCP). The proposed forward model was validated using an analytical solution. Conjugate gradient method with adjoint equation (CGMAE) was chosen as the preferred solution technique. Initially, various computational test cases were considered to estimate heat flux, base temperature and heat transfer coefficient. For all these cases, the accuracy of the inverse approach was evaluated using simulated measurements. After establishing computational robustness based on the simulated measurements, the investigation ventured into experimental validation. Sensitivity studies reveal the optimum parameter for the estimation and location of sensors. It was observed that deviations in results tend to increase with increase in time interval due to increased accumulation of noise. Obtained results highlight the accuracy and stability of predicted solution. The results obtained reinforce the reliability of the proposed model in predicting the desired values and opens up new possibilities in the field of estimation of unknown parameters in accordance with the combination of simple approximation model, experiments and CGM (inverse method).

\section{Nomenclature}

$\begin{array}{ll}A_{c} & \text { area of cross section } \\ d & \text { direction of descent } \\ g & \text { acceleration due to gravity } \\ h & \text { convective heat transfer coefficient } \\ M & \text { number of sensors } \\ k & \text { thermal conductivity of fin } \\ k_{f} & \text { thermal conductivity of fluid } \\ L & \text { fin length } \\ P(t) & \text { unknown transient parameter } \\ p & \text { fin perimeter } \\ N u & \text { Nusselt number } \\ q & \text { heat flux } \\ R a & \text { Rayleigh number } \\ T & \text { temperature } \\ T_{b} & \text { base temperature } \\ t & \text { time, s } \\ T_{\infty} & \text { ambient temperature } \\ x & \text { space coordinate } \\ Y & \text { measured temperature }\end{array}$

\section{Greek symbols}

$\begin{array}{ll}\alpha & \text { thermal diffusivity } \\ \beta & \text { coefficient of thermal expansion } \\ \beta_{k} & \text { step size } \\ \gamma & \text { coefficient of conjugation } \\ \epsilon & \text { stopping criterion/error } \\ \lambda & \text { Lagrange multiplier } \\ v & \text { kinematic viscosity } \\ \tau & \text { time step }\end{array}$




\section{Subscripts}

exp experimental temperature

$f \quad$ final

$i \quad$ sensor index

$m \quad$ measured location

$s \quad$ simulated temperature

\section{Superscripts}

$k \quad$ iteration number

\section{References}

[1] Colaco M J, Orlande H R and Dulikravich G S 2006 Inverse and optimization problems in heat transfer. J. Braz. Soc. Mech. Sci. Eng. 28(1): 1-24

[2] Beck J V, Blackwell B and Clair Jr. C R S 1985 Inverse Heat Conduction: Ill-Posed Problems. James Beck

[3] Beck J, Blackwell B, Haji-Sheikh A 1996 Comparison of some inverse heat conduction methods using experimental data. Int. J. Heat Mass Transf. 39(17): 3649-3657

[4] Beck J V, Blackwell B, Woodbury K A 1996 Comparison of some inverse heat conduction methods using experimental data. Int. J. Heat Mass Transf. 39: 3649-3657

[5] Jarny Y, Ozisik M, Bardon J 1991 A general optimization method using adjoint equation for solving multidimensional inverse heat conduction. Int. J. Heat Mass Transf. 34(11): 2911-2919

[6] Sawaf B 1995 Inverse heat conduction problems. Previews Heat Mass Transf. 6(21): 562-563

[7] Huang C H, Chen C W 1998 A boundary element-based inverse-problem in estimating transient boundary conditions with conjugate gradient method. Int. J. Numer. Methods Eng. 42(5): 943-965.

[8] Colaco M J, Orlande H R 1999 Comparison of different versions of the conjugate gradient method of function estimation. Numer. Heat Transf. Part A Appl. 36(2): 229-249

[9] Huang C H, Wang S P 1999 A three-dimensional inverse heat conduction problem in estimating surface heat flux by conjugate gradient method. Int. J. Heat Mass Transf. 42(18): 3387-3403

[10] Cheng C H, Chang M H 2003 A simplified conjugate-gradient method for shape identification based on thermal data. Numer. Heat Transf. Part B Fundam. 43(5): 489-507

[11] Lee H L, Chou H M, Yang Y C 2004 The function estimation in predicting heat flux of pin fins with variable heat transfer coefficients. Energy Convers. Manag. 45(11): 1749-1758

[12] Huang C H, Wu H H 2006 An inverse hyperbolic heat conduction problem in estimating surface heat flux by the conjugate gradient method. J. Phys. D Appl. Phys. 39(18): 4087

[13] Haghighi M G, Eghtesad M, Malekzadeh P, Necsulescu D 2008 Two dimensional inverse heat transfer analysis of functionally graded materials in estimating time-dependent surface heat flux. Numer. Heat Transf. Part A Appl. 54(7): 744-762

[14] Zhou J, Zhang Y, Chen J, Feng Z 2010 Inverse estimation of surface heating condition in a three-dimensional object using conjugate gradient method. Int. J. Heat Mass Transf. 53(13): 2643-2654

[15] Mohammadiun M, Rahimi A, Khazaee I 2011 Estimation of the time dependent heat flux using the temperature distribution at a point by conjugate gradient method. Int. J. Therm. Sci. 50(12): 2443-2450

[16] Su J, Hewitt G F 2004 Inverse heat conduction problem of estimating time-varying heat transfer coefficient. Numer. Heat Transf. Part A Appl. 45(8): 777-789

[17] Jarny Y 2001 Determination of heat sources and heat transfer coefficient for two-dimensional heat flow: numerical and experimental study. Int. J. Heat Mass Transf. 44(7): 1309-1322

[18] Chen W L, Yang Y C, Lee H L 2007 Inverse problem in determining convection heat transfer coefficient of an annular fin. Energy Convers. Manag. 48(4): 1081-1088

[19] Jin B 2007 Conjugate gradient method for the Robin inverse problem associated with the Laplace equation. Int. J. Numer. Methods Eng. 71(4): 433-453

[20] Wang Y, Luo X, Song Y, Xie Q 2017 Simultaneous reconstruction of the surface heat flux and the source term in 3D linear parabolic problem by modified conjugate gradient method. Math. Methods Appl. Sci. 40(8): 2847-2858

[21] Cheng L, Zhong F, Gu H, Zhang X 2016 Application of conjugate gradient method for estimation of the wall heat flux of a supersonic combustor. Int. J. Heat Mass Transf. 96: 249-255

[22] Ye J, Farge L, Andre S, Neveu A 2016 A numerical study of heat source re- construction for the advection-diffusion operator: a conjugate gradient method stabilized with SVD. Int. J. Therm. Sci. 104: 68-85

[23] Singh, K M, Yadav, D, Arpit, S, Mitra, S, Saha, K S 2016 Effect of nanofluid concentration and composition on laminar jet impinged cooling of heated steel plate. Appl. Therm. Eng. 100: 237-246

[24] Zhang D D, Zhang J H, Liu D, Zhao F Y, Wang H Q, Li X H 2016 Inverse conjugate heat conduction and natural convection inside an enclosure with multiple unknown wall heating fluxes. Int. J. Heat Mass Transf. 96: 312-329

[25] Ozisik M N, Orlande H R B 2000 Inverse Heat Transfer: Fundamentals and Applications. CRC Press, Boca Raton

[26] Beck J V, Woodbury K A. 2016 Inverse heat conduction problem: sensitivity coefficient insights, filter coefficients, and intrinsic verification. Int. J. Heat Mass Transf. 97: 578-588

[27] Kern D Q, Kraus A D 1972 Extended Surface Heat Transfer. MCGrawHill, New York

[28] Venugopal G, Deiveegan M, Balaji C, Venkateshan S 2008 Simultaneous retrieval of total hemispherical emissivity and specific heat from transient multimode heat transfer experiments. J. Heat Transf. 130(6): 061601

[29] Gnanasekaran N, Balaji C 2010 An inexpensive technique to simultaneously determine total emissivity and natural convection heat transfer coefficient from transient experiments. Exp. Heat Transf. 23(3): 235-258 